\title{
miR-92b targets DAB2IP to promote EMT in bladder cancer migration and invasion
}

\author{
JUN HUANG $^{1}$, BIN WANG $^{1}$, KE HUI $^{1}$, JIN ZENG $^{1}$, JINHAI FAN $^{1}$, \\ XINYANG WANG ${ }^{1}$, JER-TSONG HSIEH ${ }^{2}$, DALIN HE ${ }^{1}$ and KAIJIE WU ${ }^{1}$ \\ ${ }^{1}$ Department of Urology, The First Affiliated Hospital of Xi'an Jiaotong University, Xi'an, Shaanxi 710061, P.R. China; \\ ${ }^{2}$ Department of Urology, University of Texas, Southwestern Medical Center, Dallas, TX 75390, USA
}

Received March 11, 2016; Accepted July 7, 2016

DOI: $10.3892 /$ or.2016.4940

\begin{abstract}
Muscle-invasive or metastatic bladder cancer (BCa) has a very poor prognosis; however, its mechanisms remain largely unknown. Previous studies have discovered multiple microRNAs (miRs) that are involved in $\mathrm{BCa}$ progression and regarded as potential biomarkers or therapeutic targets. In this study, we demonstrated that miR-92b could uniquely promote cell migration and invasion of BCa cells, but had no effect on cell proliferation. Mechanistically, our data provided evidence to verify that miR-92b was able to directly target DAB2IP, a well-known tumor suppressor, and inhibit epithelial-mesenchymal transition of BCa cells. Moreover, the increased expression levels of miR-92b were negatively correlated with DAB2IP, and predicted poor prognosis of patients with $\mathrm{BCa}$. Overall, this study reveals a new promising biomarker and its mechanisms contributing to $\mathrm{BCa}$ invasion or metastasis.
\end{abstract}

\section{Introduction}

Bladder cancer $(\mathrm{BCa})$ is one of the most common malignant diseases around the world. In the United States, the estimated new cases in 2015 were 74,000, and the deaths caused by BCa about 16,000. Statistical data from 2004 to 2010 in the United States showed that, the five-year survival rate for localized $\mathrm{BCa}$ was $69 \%$, for regional $\mathrm{BCa} 34 \%$, but for distant $\mathrm{BCa}$ only $6 \%$, which means distant metastasis is the main cause of death in $\mathrm{BCa}$ (1). Thus, further understanding of the mechanism leading to $\mathrm{BCa}$ invasion and metastasis is still needed.

MicroRNAs (miRs) are single-strand small non-coding RNAs which take part in different processes of biological eval-

Correspondence to: Dr Dalin He or Dr Kaijie Wu, Department of Urology, The First Affiliated Hospital of Xi'an Jiaotong University, 277 Yanta West Road, Xi'an, Shaanxi 710061, P.R. China

E-mail: dalinhe@yahoo.com

E-mail:kaijie_wu@163.com

Key words: microRNA-92b, DAB2IP, bladder cancer, epithelialmesenchymal transition uation. During cancer progression, miRs not only affect cancer cell behavior such as proliferation, migration and invasion, but are also used to predict the prognosis of patients. A growing number of studies have discovered the dysregulated expression of miRs and the underlying mechanisms in $\mathrm{BCa}$ invasion or metastasis (2). For example, our previous study demonstrated that tumor-suppressive miR-145 could inhibit the invasion of BCa cells by targeting P21-activated kinase 1 (PAK1) (3). On the other hand, another miR, miR-92b, acts as an oncogene in certain malignancies including glioma, non-small cell lung cancer, and oral squamous cell carcinoma (4-8); however, its role in $\mathrm{BCa}$ remains poorly understood.

DAB2IP, a member of the RAS-GTPase-activating protein (RAS-GAP) family (9), is downregulated in multiple cancer types, including $\mathrm{BCa}$. Our and other previous studies have shown that DAB2IP loss in BCa tissues is not only associated with the intravesical pirarubicin chemoresistance, but also facilitates tumor invasion and metastasis $(10,11)$. More importantly, DAB2IP may be a promising biomarker to predict the prognosis of patients with $\mathrm{BCa}$ (12). However, the mechanism of DAB2IP downregulation in $\mathrm{BCa}$ is not yet clarified.

In this study, we demonstrated that miR-92b could significantly promote cell migration and invasion of the BCa cell line 5637, but had no effect on cell proliferation. Furthermore, our data provided evidence to verify that miR-92b could inhibit epithelial-mesenchymal transition (EMT) of $\mathrm{BCa}$ cells. Mechanistically, we showed that the tumor suppressor, DAB2IP, is a direct target of miR-92b. Indeed, restoration of DAB2IP expression could reverse all these biological effects of miR-92b in BCa cells. Moreover, we analyzed The Cancer Genome Atlas (TCGA) database and found that the expression level of miR-92b in BCa tissues was higher than normal, and there was a negative correlation between miR-92b level and DAB2IP expression. Importantly, miR-92b upregulation alone or combination with DAB2IP downregulation could predict a lower recurrence-free survival (RFS) of patients with BCa. Taken together, these results suggest that miR-92b acts as a critical onco-miR in $\mathrm{BCa}$ progression.

\section{Materials and methods}

Cell culture and transfection. The BCa cell line 5637 and human embryonic kidneys (HEK) cell line 293T were main- 
Table I. Primers and sequences used in this study.

\begin{tabular}{lll}
\hline Genes & \multicolumn{1}{c}{ Forward primers (5'-3') } & \multicolumn{1}{c}{ Reverse primers (5'-3') } \\
\hline GAPDH & ATGGGGAAGGTGAAGGTCGG & GACGGTGCCATGGAATTTGC \\
CDH1 & TGCCCAGAAAATGAAAAAGG & GTGTATGTGGCAATGCGTTC \\
CDH2 & ACAGTGGCCACCTACAAAGG & CCGAGATGGGGTTGATAATG \\
VIM & GAGAACTTTGCCGTTGAAGC & GCTTCCTGTAGGTGGCAATC \\
MMP2 & GATACCCCTTTGACGGTAAGGA & CCTTCTCCCAAGGTCCATAGC \\
MMP9 & CATTTCGACGATGACGAGTTGT & CGGGTGTAGAGTCTCTCGC \\
SNAI1 & GACCACTATGCCGCGCTCTT & TCGCTGTAGTTAGGCTTCCGATT \\
SNAI2 & TTTCCAGACCCTGGTTGCTTC & CTCAGATTTGACCTGTCTGCAAATG \\
ZEB1 & GAAAGTGATCCAGCCAAATGGAA & TTTGGGCGGTGTAGAATCAGAG \\
ZEB2 & AAATGCACAGAGTGTGGCAAGG & CTGCTGATGTGCGAACTGTAGGA \\
TWIST1 & GTCCGCAGTCTTACGAGGAG & GCTTGAGGGTCTGAATCTTGCT
\end{tabular}

tained in the Institute of Urology, Xi'an Jiaotong University. The 5637 cells were cultured in RPMI-1640 and 293T were cultured in Dulbecco's modified Eagle's medium (DMEM) with $10 \%$ fetal bovine serum (FBS) at $37^{\circ} \mathrm{C}$ with $5 \% \mathrm{CO}_{2}$.

miR-92b mimic, miR-negative control (miR-NC) mimic, miR-92b inhibitor and miR-NC inhibitor were purchased from Guangzhou RiboBio Co., Ltd. (Guangzhou, China). DAB2IP cDNA and its vector, DAB2IP shRNA and its scrambled shRNA have been described in our previous studies (11). Cells were transfected using Lipofectamine 2000 (Invitrogen, Carlsbad, CA, USA) according to the manufacturer's instructions.

Real-time quantitative PCR (RT-qPCR). Total RNA was isolated from cells using TRIzol reagent (Life Technologies, Rockville, MD, USA). For miRNA RT-qPCR, miScript II RT kit (Qiagen, Valencia, CA, USA) was used for reverse transcription, and U6 was used as an internal control. The primer sequence for miR-92b was 5'-TAT TGC ACT CGT CCC GGC CTC C-3' [Tiangen Biotech (Beijing) Co., Ltd., Beijing, China]. Reverse transcription for other genes was performed with PrimeScript ${ }^{\mathrm{TM}}$ RT reagent kit (Takara, Dalian, China). CFX96 Real-Time PCR system (Bio-Rad Laboratories, Inc., Hercules, CA, USA) with SYBR-Green PCR Master Mix (Takara) was used to determine the different gene expression levels. GAPDH was used as another internal control. Primer sequences used in this study are shown in Table I.

Wound healing assay. Wounds were scratched using a 200- $\mu 1$ pipette tips when transfected cells in six-well plate were grown to $100 \%$, and the culture medium was changed to serum-free medium. The wound closure was monitored by inverted microscope after $24 \mathrm{~h}$.

Transwell migration and invasion assay. The 5637 cells were digested $24 \mathrm{~h}$ later after transfection. For migration assay, $5 \times 10^{4}$ cells in $200 \mu \mathrm{l}$ serum-free medium were seeded in the upper chamber of the Transwell without Matrigel. For invasion assay, $1.2 \times 10^{5}$ cells in $200 \mu \mathrm{l}$ serum-free medium were seeded in the upper chamber of the Transwell with Matrigel. The lower chamber was filled with $600 \mu 1$ medium with $20 \%$
FBS. After 24 -h incubation at $37^{\circ} \mathrm{C}$ with $5 \% \mathrm{CO}_{2}$, cells were fixed with $4 \%$ paraformaldehyde and stained with $0.1 \%$ crystal violet. The number of migrated or invaded cells was counted using a microscope in three random fields.

3-(4,5-Dimethylthiazol-2-yl)-2,5-diphenyltetrazolium bromide (MTT) assay. The 5637 cells were digested $24 \mathrm{~h}$ after transfection, and $2 \times 10^{3}$ cells in $200 \mu$ l complete medium were seeded into 96-well plates/well, then the cells were incubated for 24, 48,72 and $96 \mathrm{~h}$ before $20 \mu \mathrm{l}$ MTT was added to each well and incubated for another $4 \mathrm{~h}$ in $37^{\circ} \mathrm{C}$. Then the medium was discared and $150 \mu \mathrm{l}$ DMSO was added, after $10 \mathrm{~min}$ of shaking, the absorbance of $490 \mathrm{~nm}$ was measured using the microplate autoreader (Bio-Tek Instruments, Winooski, VT, USA).

Western blot analysis. Cells were washed with cold PBS and then lysed in RIPA buffer containing protease inhibitor. Then total protein was loaded into 10\% SDS-PAGE gel and blotted onto nitrocellulose membranes. The membrane was blocked with 5\% skim milk for $1 \mathrm{~h}$, then incubated with primary antibodies against DAB2IP (Abcam, Cambridge, UK), E-cadherin, N-cadherin, vimentin, matrix metalloproteinase (MMP) 2 and MMP9 (Santa Cruz Biotechnology, Inc. Santa Cruz, CA, USA) overnight at $4^{\circ} \mathrm{C}$. After washing with TBST three times, the membrane was incubated with horseradish peroxidase-conjugated secondary antibodies at room temperature for $1 \mathrm{~h}$. Then the bands were visualized by ECL system (Bio-Rad Laboratories, Inc.). Loading differences were normalized using a monoclonal GAPDH antibody (KangChen Bio-tech, Inc., Shanghai, China).

Dual-luciferase reporter assay. Wild-type (WT) and mutation (MUT) of DAB2IP 3'-UTR reporter plasmids were designed and obtained from GenePharma (Shanghai, China). To perform the dual-luciferase reporter assay, WT or MUT DAB2IP 3'-UTR plasmids were transfected into 293T cells with miR-92b mimic or inhibitor together using Lipofectamine 2000. After 24-h incubation, luciferase assay was carried out using the dual-luciferase assay kit (Promega, Madison, WI, USA) according to the manufacturer's instructions. 
A
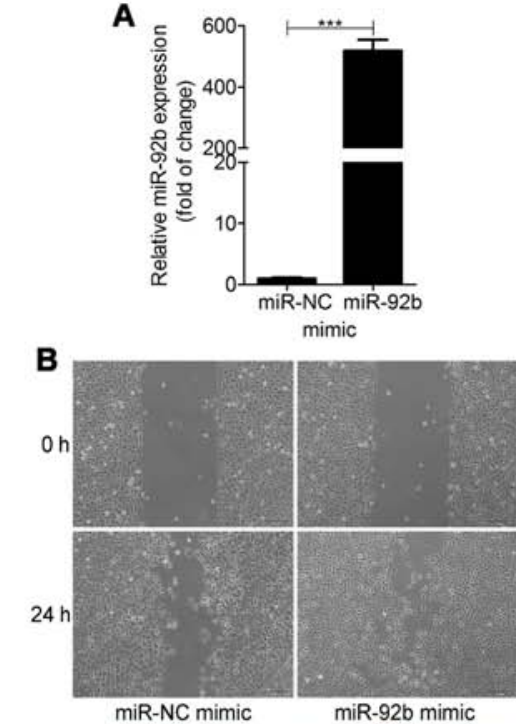

C
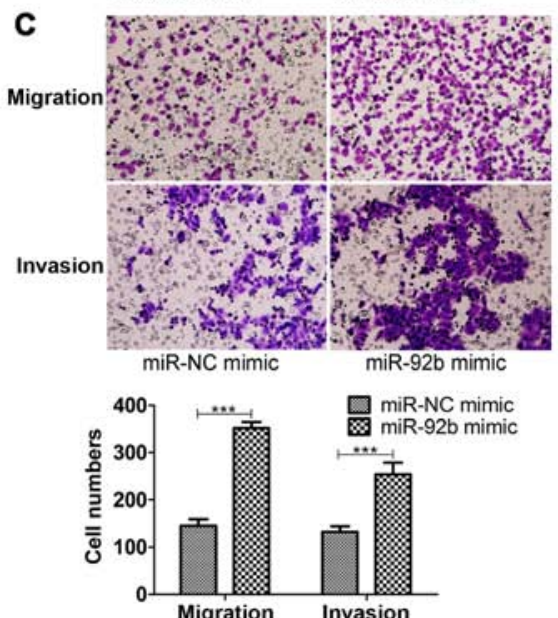

D

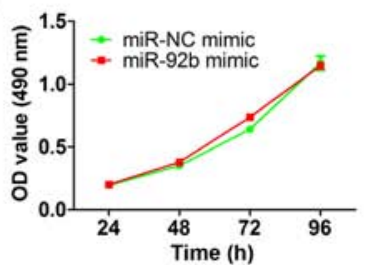

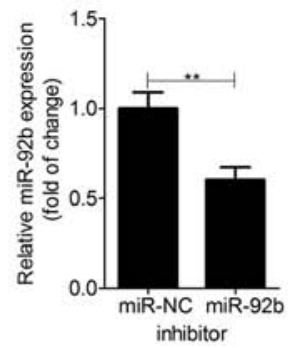
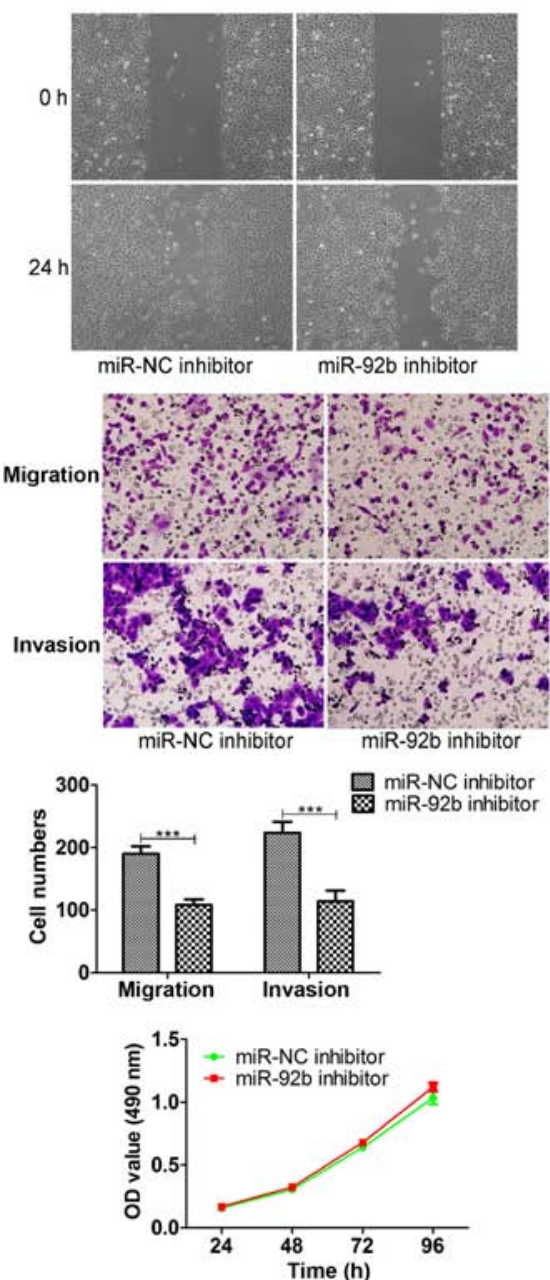

Figure 1. miR-92b promotes 5637 cell migration and invasion in vitro. (A) Expression levels of miR-92b in the 5637 cells after transfection with miR-92b mimic (left panel) or inhibitor (right panel) determined by RT-qPCR. ${ }^{* * *} \mathrm{P}<0.001$ vs. miR-negative control (miR-NC). (B) representative pictures showing the wound healing of the 5637 cells after transfection with miR-92b mimic (left panel) or inhibitor (right panel). (C) Representative pictures of Transwell migration and invasion assays showing the migration and invasion abilities of the 5637 cells after transfection with miR-92b mimic (left panel) or inhibitor (right panel). Quantification analysis is shown, ${ }^{* * *} \mathrm{P}<0.001$ vs. miR-NC. (D) The 5637 were transfected with miR-92b mimic (left panel) or inhibitor (right panel), and then subjected to cell viability assay.

Bioinformatics. miRNA expression and gene expression data in BCa tissues were downloaded from TCGA database (https://genome-cancer.ucsc.edu/proj/site/hgHeatmap/) at February 24,2015 , the data files were used to analyze the expression levels of miR-92b, DAB2IP, and their correlation with the overall survival (OS) and RFS of patients.

Statistical analysis. GraphPad Prism version 6.0 software (GraphPad Software, Inc., La Jolla, CA, USA) was used to analyze the difference between experimental groups. Quantitative data are presented as mean $\pm \mathrm{SD}$, and the differences between two groups were compared by the two-tailed
Student's t-test. A P $<0.05$ was considered to be statistically significant.

\section{Results}

miR-92b promotes $B C$ a cell migration and invasion in vitro. To explore the role of miR-92b in $\mathrm{BCa}$, we transfected the $\mathrm{BCa}$ cell line 5637 with miR-92b mimic or inhibitor and examined its biological function in BCa. As showed in Fig. 1A, RT-qPCR assay detected the change of miR-92b expression in the 5637 cells $48 \mathrm{~h}$ after transfection. Subsequently, miR-92b upregulation could significantly enhance the wound healing 

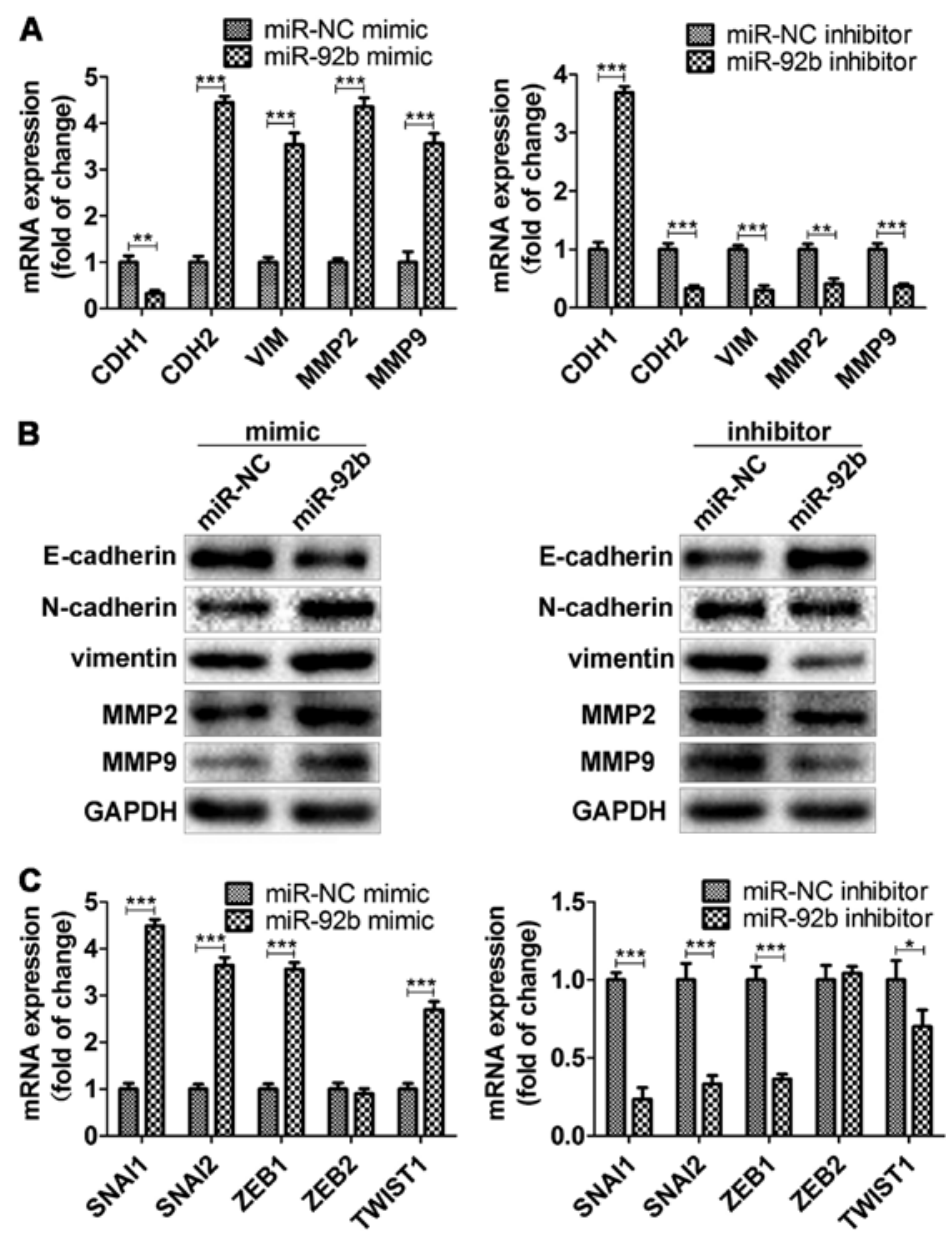

Figure 2. miR-92b promotes epithelial-mesenchymal transition (EMT) in the 5637 cells. (A) CDH1 (E-cadherin), CDH2 (N-cadherin), VIM (vimentin), matrix metalloproteinase (MMP) 2 and MMP9 mRNA were detected in the 5637 cells transfected with miR-92b mimic (left panel) or inhibitor (right panel). The relative mRNA level of each gene was determined by normalizing to GAPDH. Results (mean \pm SD) were obtained from three independent experiments. ${ }^{* * * * *} \mathrm{P}<0.001$ vs. miR-negative control (miR-NC). (B) E-cadherin, N-cadherin, vimentin, MMP2 and MMP9 proteins were detected in the 5637 cells transfected with miR-92b mimic (left panel) or inhibitor (right panel) by western blot analysis, and GAPDH was used as a loading control. (C) SNAI1, SNAI2, ZEB1, ZEB2 and TWIST1 mRNA were detected in the 5637 cells transfected with miR-92b mimic (left panel) or inhibitor (right panel). The relative mRNA level of each gene was determined by normalizing to GAPDH. Results (mean $\pm \mathrm{SD}$ ) were obtained from three independent experiments. ${ }^{* * *} \mathrm{P}<0.001 \mathrm{vs}$. miR-NC. ${ }^{*} \mathrm{P}<0.05$ vs. miR-NC.

rate; whereas, miR-92b inhibitor decreased this rate (Fig. 1B). Moreover, the Transwell migration and invasion assay further proved that miR-92b promoted the ability of migration and invasion in 5637 cells, but downregulation of miR-92b abolished cell migration and invasion (Fig. 1C; $\mathrm{P}<0.001$ ). However, similar treatment with miR-92b mimic or inhibitor had no effect on cell proliferation by MTT assays (Fig. 1D), indicating a unique role of miR-92b in BCa invasion and metastasis, but not tumor growth.

miR-92b promotes EMT in BCa cells. EMT is well-known to play an important role in tumor invasion and metastasis by endowing cells with a more motile and invasive phenotype $(13,14)$. Both transcriptional and post-transcriptional regulatory mechanisms contribute to this complex network during the EMT process, and substantil evidence demonstrates that miRs are crucial regulators of this network (15). Herein, we further demonstrated that miR-92b could induce EMT of the 5637 cells. RT-qPCR and western blot results showed that miR-92b upregulation suppressed the expression of epithelial marker E-cadherin, but increased the expression of mesen- chymal markers including N-cadherin, vimentin, and MMP family proteins such as MMP2 and MMP9 (Fig. 2A and B, left panel; $\mathrm{P}<0.001)$. Instead, miR-92b inhibitor presented the opposite results (Fig. 2A and B, right panel; $\mathrm{P}<0.001$ ). Furthermore, we observed a different effect of miR-92b on the expression of crucial EMT-related transcription factors, such as Snail, ZEB and Twist families. For example, RT-qPCR assay showed that SNAI1, SNAI2, ZEB1 and TWIST1 expression were significantly upregulated by miR-92b (Fig. 2C; $\mathrm{P}<0.001, \mathrm{P}<0.05$ ). These results indicate that miR-92b could regulate cell migration and invasion by inducing EMT in $\mathrm{BCa}$.

$D A B 2 I P$ is a direct target of miR-92b. Mechanically, miR works mainly by binding to the 3'-UTR (un-translated region) of its target mRNA, by which miR degrades the mRNA or suppresses its translation. To elucidate the target gene of miR-92b in BCa, we searched TargetScan and PicTar, and both tools predicted DAB2IP as a potential target of miR-92b. RT-qPCR and western blot assays were performed, and demonstrated that miR-92b downregulated both DAB2IP mRNA and protein levels (Fig. 3A and B). Also, by co-transfection the 
B
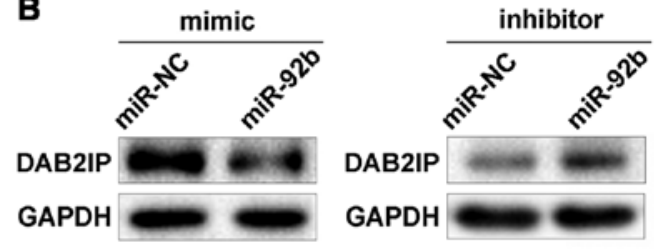

DAB2IP 3'UTR WT 5' AGACUCUGUGUGGCUGUGCAAUU DAB2IP 3'UTR MUT 5' AGACUCUGUGUGGCUCACGUAUU
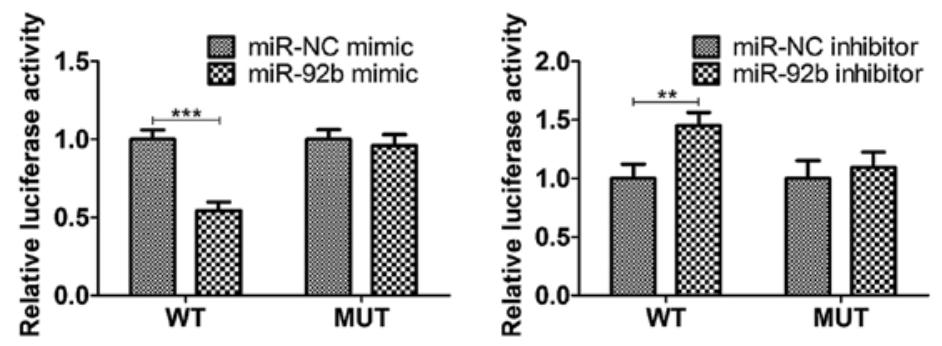

Figure 3. DAB2IP is a direct target of miR-92b in bladder cancer (BCa) cells. (A) DAB2IP mRNA was detected in the 5637 cells transfected with miR-92b mimic (left panel) or inhibitor (right panel). The relative mRNA level of each gene was determined by normalizing to GAPDH. Results (mean \pm SD) were obtained from three independent experiments. ${ }^{* * *} \mathrm{P}<0.001 \mathrm{vs}$. miR-negative control (miR-NC). ${ }^{*} \mathrm{P}<0.01 \mathrm{vs}$. miR-NC. (B) DAB2IP protein was detected in the 5637 cells transfected with miR-92b mimic (left panel) or inhibitor (right panel) by western blot analysis, and GAPDH was used as a loading control. (C) The potential miR-92b binding sites of wild-type (WT) DAB2IP 3'UTR and the mutated sequences are shown (upper panel). The relative luciferase activity was detected in 293T cells co-transfected with miR-92b mimic (or inhibitor) and reporter plasmid carrying WT or mutant DAB2IP 3'UTR (lower panel); data are shown as mean $\pm \mathrm{SD} ;{ }^{* * *} \mathrm{P}<0.001$ vs. miR-NC. ${ }^{*} \mathrm{P}<0.05$ vs. miR-NC.

reporter plasmid carrying WT DAB2IP 3'-UTR with miR-92b mimic or inhibitor in 293T cells, we found that miR-92b significantly reduced its luciferase activity. In contrast, this suppressive effect was abolished when the miRNA binding sequence in DAB2IP 3'-UTR was mutated (Fig. 3C). These results suggest that DAB2IP is a direct target of miR-92b.

DAB2IP mediates the biological effects of miR-92b in BCa cells. To determine whether DAB2IP is critical for cell migration and invasion of BCa cells by miR-92b, we restored or knocked down DAB2IP expression in miR-92b mimic- or inhibitortransfected 5637 cells. Indeed, as shown in Fig. 4A, DAB2IP overexpression could abolish the increased wound healing rate induced by miR-92b in the 5637 cells. In contrast, knockdown of DAB2IP by shRNA restored the ability of wound healing in the 5637 cells transfected with miR-92b inhibitor. Moreover, Transwell migration and invasion assays showed similar results after manipulation of DAB2IP expression levels (Fig. 4B and C; $\mathrm{P}<0.001, \mathrm{P}<0.01$ and $\mathrm{P}<0.05$ ). Thus, these data demonstrate that DAB2IP mediates the biological functions of miR-92b in $\mathrm{BCa}$ as an important downstream target.

$D A B 2 I P$ reverses the phenotype of EMT induced by miR-92b. Our recent studies have discovered the tumor-suppressive roles of DAB2IP in the regulation of cell apoptosis, survival (16), autophagy (17), DNA repair (18), EMT (19) and cancer stem cells (CSCs) (20), all of which may contribute to the tumor initiation and progression. Indeed, as shown in Fig. 5, restoration of DAB2IP in miR-92b mimic-transfected 5637 cells dramatically increased E-cadherin expression, but decreased vimentin and MMP9 expression, however, knockdown of DAB2IP in miR-92b inhibitor-transfected 5637 cells resulted in a decrease of E-cadherin expression but an increase of vimentin and MMP9 expression. These data support that miR-92b could target DAB2IP and induce EMT for $\mathrm{BCa}$ migration and invasion.

Expression and clinical significance of miR-92b in patients with $B C a$. To evaluate the role of miR-92b in clinic, we searched the TCGA database to analyze its expression and clinical significance. First, we found 19 paired $\mathrm{BCa}$ and adjacent normal tissues with available miR-92b and DAB2IP expression data. As shown in Fig. 6A, the expression of miR-92b was higher in $\mathrm{BCa}$ tissues than normal, while the expression of DAB2IP is significantly lower in BCa tissues. Also, there was a negative correlation between miR-92b and DAB2IP expression. Importantly, we further observed a significant association between miR-92b level and RFS of patients with $\mathrm{BCa}$. Although there was no statistical correlation between miR-92b expression levels and the OS of patients with BCa (Fig. 6B and C, left panel), miR-92b upregulation alone or combination with DAB2IP downregulation could specifically predict a lower RFS of patients (Fig. 6B and C, right panel). These clinical data suggest miR-92b and DAB2IP as good prognostic markers for patients with $\mathrm{BCa}$.

\section{Discussion}

$\mathrm{BCa}$ is a major cause of morbidity and mortality worldwide, in which muscle-invasive BCa (MIBC) with a high frequency of 
A

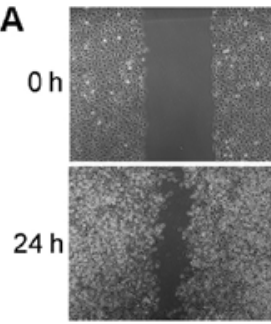

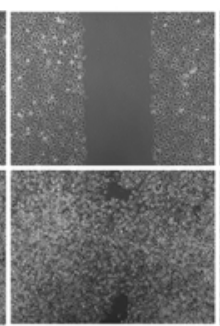

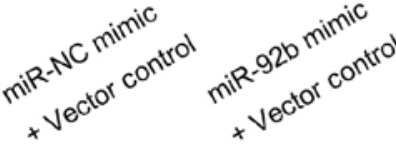

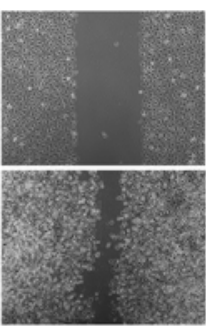

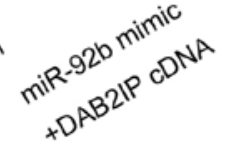

B
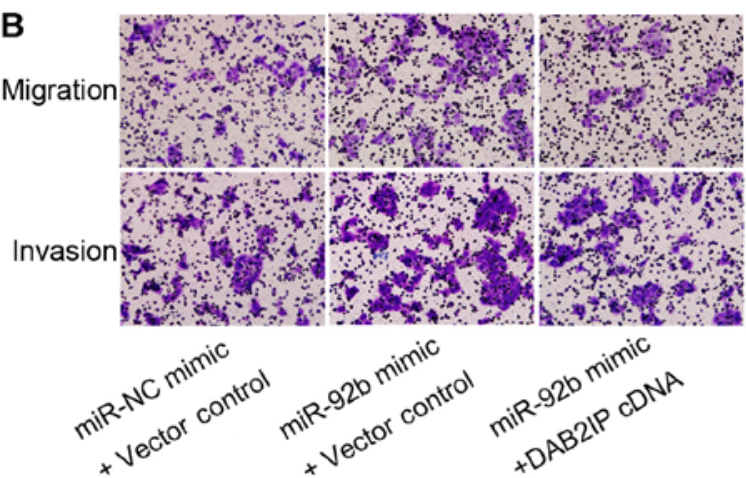

\section{C miR-NC mimic + Vector control \% miR-92b mimic + Vector contro miR-92b mimic + DAB2IP cDNA}
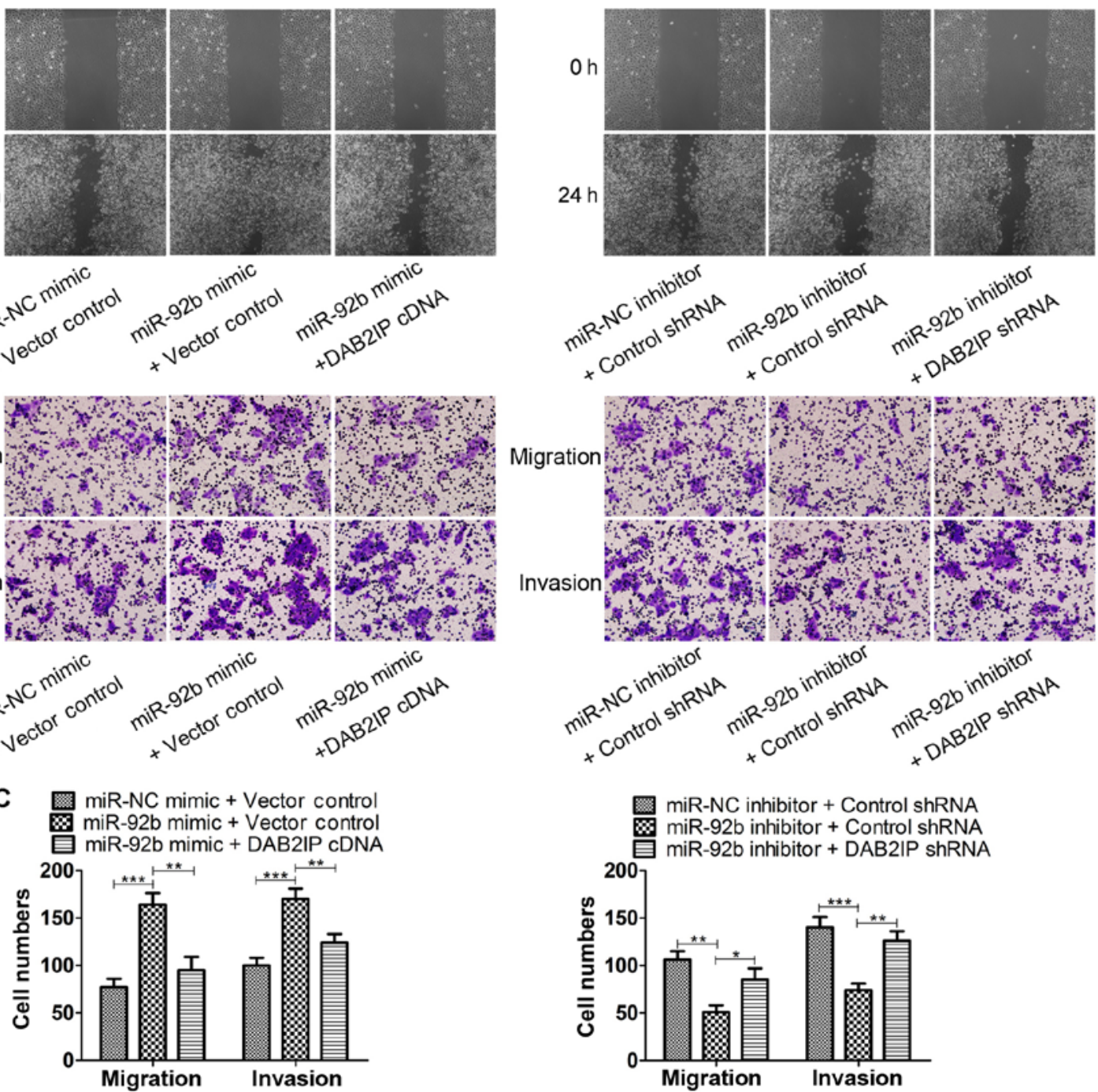

Figure 4. DAB2IP abolishes the biological effects of miR-92b in bladder cancer (BCa) cells. (A) Representative pictures showing the wound healing of 5637 cells co-transfected with miR-92b mimic (or inhibitor) and DAB2IP cDNA (or shRNA). (B) Representative pictures of Transwell migration and invasion assays showing the migration and invasion abilities of the 5637 cells co-transfected with miR-92b mimic (or inhibitor) and DAB2IP cDNA (or shRNA). (C) Quantification analysis of Transwell migration and invasion assays is shown, ${ }^{* * *} \mathrm{P}<0.001$ vs. miR-NC. ${ }^{* *} \mathrm{P}<0.01$ as indicated.
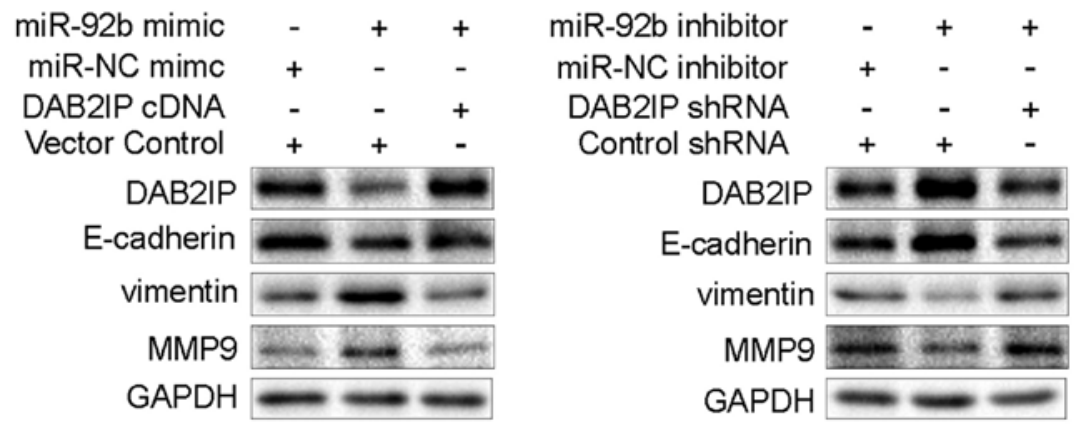

Figure 5. DAB2IP abolishes epithelial-mesenchymal transition (EMT) induced by miR-92b. The 5637 cells were co-transfected with miR-92b mimic (or inhibitor) and DAB2IP cDNA (or shRNA), and then DAB2IP, E-cadherin, N-cadherin, vimentin and MMP9 proteins were detected by western blot analysis. GAPDH was used as a loading control.

metastasis has very poor prognosis for patients. Understanding and clarifying these mechanisms of cancer metastasis will help to develop new therapeutic strategies and improve patient survival. Recently, several whole-genome studies provided a comprehensive landscape of molecular alterations in $\mathrm{BCa}$, especially intrinsic basal and luminal MIBC subtypes associated with different chemotherapy sensitivity or prognosis were well characterized $(21,22)$. In our previous studies, we developed unique isogenetic T24-t sublines and discovered a complex regulatory network contributing to $\mathrm{BCa}$ metastasis. 

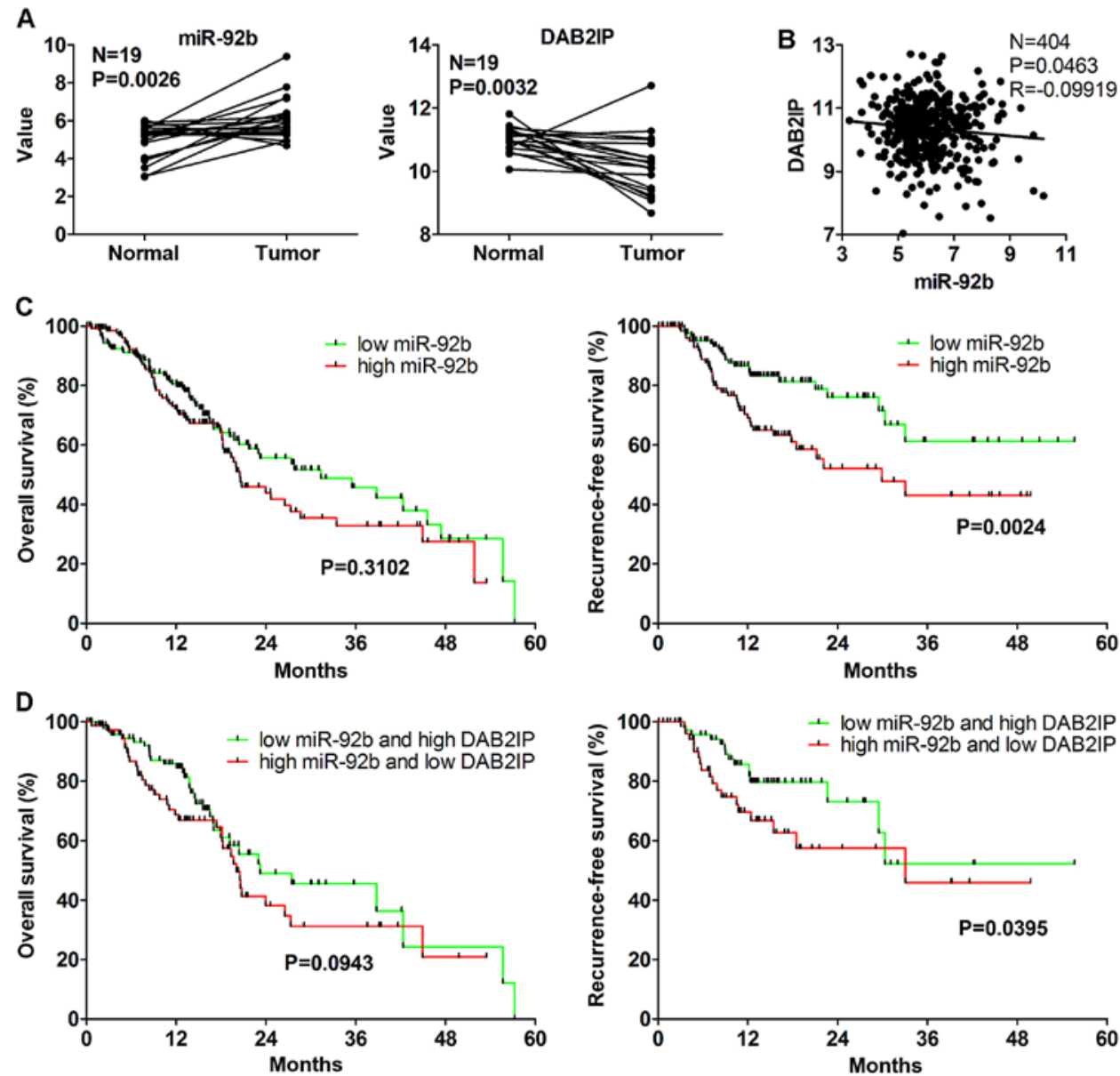

Figure 6. Correlation between miR-92b and DAB2IP in human bladder cancer (BCa) tissues and its significance for patient prognosis. (A) Analysis of miR-92b and DAB2IP expression in 19 paired BCa and adjacent normal tissues from a public microarray database [The Cancer Genome Atlas (TCGA)]. (B) Correlation between miR-92b and DAB2IP mRNA in BCa tissues from TCGA $(n=404)$. (C and D) Percentage of overall survival and recurrence-free survival was analyzed by Kaplan-Meier curves from the TCGA database.

For example, HIF-1 $\alpha$-regulated MMP-1 expression, Slug-

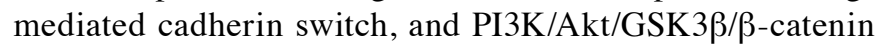
signaling cascade-initiated ZEB1 transcription all endowed $\mathrm{BCa}$ cells with the mesenchymal and CSC-like properties (23-25). In the present study, we further explore the roles of miRs, another crucial regulator in this network, and first provide the evidence to show that miR-92b induces EMT in BCa.

miRs have been reported to play critical roles in different cancer progression, including BCa. For example, miR-100 targeted the fibroblast growth factor receptor 3 (FGFR3) to maintain $\mathrm{BCa}$ cell viability under conditions of hypoxia stress (26). Our previous study also demonstrated that the tumor suppressive miR-145 inhibited invasion of BCa cells via targeting PAK1 (3). Herein, we reported the function of another important miR, miR-92b, in BCa migration and invasion via targeting DAB2IP.

DAB2IP, also named ASK1-interacting protein 1 (AIP1), acts as a crucial tumor suppressor in different human malignancies, such as prostate cancer (27), breast cancer (28), lung cancer (29), gastrointestinal tumor (30), liver cancer (31), pancreatic cancer (32), medulloblastoma (33) and esophageal carcinoma (34). It plays vital roles in different aspects of biological activities, including cell apoptosis or survival (16), autophagy (17), DNA repair (18), EMT (19) and CSC (20), all of which may contribute to the tumor initiation, growth, metastasis and therapeutic resistance. Until now, we have known that DAB2IP gene transcription is usually regulated by epigenetic mechanisms, such as DNA methylation and histone modification (35), Also, DAB2IP expression could be modulated by Skp2-mediated proteasome degradation (36). Recently, several studies demonstrated that different miRs could target DAB2IP 3'-UTR and suppress its expression. For example, $\mathrm{Xu}$ et al reported that miR-889 promoted proliferation of esophageal squamous cell carcinomas through DAB2IP (34), while another study showed that miR-32 induced radioresistance by targeting DAB2IP and regulating autophagy in prostate cancer (37). In this study, we further revealed that DAB2IP could also be targeted by miR-92b in $\mathrm{BCa}$ and facilitated EMT for tumor cell migration or invasion, indicating a complex upstream regulation for DAB2IP in different cancer types.

The importance of miR-92b in cancer development has been established by other studies. In neuroblastoma, miR-92b could be regulated by MYCN, which then inhibits secretion of the tumor suppressor DKK3 (38). By directly targeting Nemo-like kinase and DKK3, miR-92b controls glioma cell proliferation, invasion and apoptosis (6). In non-small cell lung cancer, miR-92b targets PTEN and RECK to regulate cell growth and cisplatin chemosensitivity (39). Also, 
miR-92b functions as a potential oncogene by targeting Smad3 in glioblastomas (5). Moreover, in hepatic progenitors, aberrant expression of miR-92b is associated with proliferation increase and differentiation arrest (40). We are the first to show the role of miR-92b in BCa cells based on both gain-of-function and loss-of-function strategies. In addition, our analysis of clinical data from TCGA database also showed the increased miR-92b levels along with decreased DAB2IP expression in BCa specimens predicted a poor RFS rate of patients, which indicates that miR-92b may be another good prognotic molecular marker for patients with $\mathrm{BCa}$ in future.

Taken together, our studies revealed the critical role of miR-92b in modulating migration and invasion of $\mathrm{BCa}$ cells in vitro, in which its direct downstream target gene DAB2IP regulates the EMT phenotype. Importantly, our findings provide a molecular and clinical basis highlighting miR-92b as a potential biomarker in predicting the outcome of BCa.

\section{Acknowledgements}

This study was supported by the National Natural Science Foundation of China (NSFC 81572516 awarded to K.W.; NSFC 81572520 awarded to J.F.).

\section{References}

1. Siegel RL, Miller KD and Jemal A: Cancer statistics, 2015. CA Cancer J Clin 65: 5-29, 2015

2. Shi Z, Wei Q, Zhang M and She J: MicroRNAs in bladder cancer: Expression profiles, biological functions, regulation, and clinical implications. Crit Rev Eukaryot Gene Expr 24: 55-75, 2014.

3. Kou B, Gao Y, Du C, Shi Q, Xu S, Wang CQ, Wang X, He D and Guo P: miR-145 inhibits invasion of bladder cancer cells by targeting PAK1. Urol Oncol 32: 846-854, 2014

4. Wang K, Wang X, Zou J, Zhang A, Wan Y, Pu P, Song Z, Qian C, Chen Y, Yang S, et al: miR-92b controls glioma proliferation and invasion through regulating $\mathrm{Wnt} / \mathrm{beta}$-catenin signaling via Nemo-like kinase. Neuro-oncol 15: 578-588, 2013.

5. Wu ZB, Cai L, Lin SJ, Lu JL, Yao Y and Zhou LF: The miR-92b functions as a potential oncogene by targeting on Smad3 in glioblastomas. Brain Res 1529: 16-25, 2013.

6. Li Q, Shen K, Zhao Y, Ma C, Liu J and Ma J: MiR-92b inhibitor promoted glioma cell apoptosis via targeting DKK3 and blocking the Wnt/beta-catenin signaling pathway. J Transl Med 11: $302,2013$.

7. Lei L, Huang Y and Gong W: Inhibition of miR-92b suppresses nonsmall cell lung cancer cells growth and motility by targeting RECK. Mol Cell Biochem 387: 171-176, 2014.

8. Liu Z, Diep C, Mao T, Huang L, Merrill R, Zhang Z and Peng Y: MicroRNA-92b promotes tumor growth and activation of NF- $\kappa B$ signaling via regulation of NLK in oral squamous cell carcinoma. Oncol Rep 34: 2961-2968, 2015.

9. Chen H, Toyooka S, Gazdar AF and Hsieh JT: Epigenetic regulation of a novel tumor suppressor gene (hDAB2IP) in prostate cancer cell lines. J Biol Chem 278: 3121-3130, 2003.

10. Shen YJ, Kong ZL, Wan FN, Wang HK, Bian XJ, Gan HL, Wang CF and Ye DW: Downregulation of DAB2IP results in cell proliferation and invasion and contributes to unfavorable outcomes in bladder cancer. Cancer Sci 105: 704-712, 2014.

11. Wu K, Wang B, Chen Y, Zhou J, Huang J, Hui K, Zeng J, Zhu J, Zhang K, Li L, et al: DAB2IP regulates the chemoresistance to pirarubicin and tumor recurrence of non-muscle invasive bladder cancer through STAT3/Twist1/P-glycoprotein signaling. Cell Signal 27: 2515-2523, 2015.

12. Zhu JN, Wu KJ, Guan ZF, Liu LX, Ning ZY, Zhou JC, Wang XY and Fan JH: DAB2IP expression in bladder transitional cell carcinoma and its correlation with clinical outcome. Sichuan Da Xue Xue Bao Yi Xue Ban 45: 591-594, 2014 (In Chinese).
13. McConkey DJ, Choi W, Marquis L, Martin F, Williams MB, Shah J, Svatek R, Das A, Adam L, Kamat A, et al: Role of epithelial-to-mesenchymal transition (EMT) in drug sensitivity and metastasis in bladder cancer. Cancer Metastasis Rev 28: 335-344, 2009.

14. Thiery JP, Acloque H, Huang RY and Nieto MA: Epithelial-mesenchymal transitions in development and disease. Cell 139: 871-890, 2009.

15. Zhang J and Ma L: MicroRNA control of epithelial-mesenchymal transition and metastasis. Cancer Metastasis Rev 31: 653-662, 2012.

16. Xie D, Gore C, Zhou J, Pong RC, Zhang H, Yu L, Vessella RL, Min W and Hsieh JT: DAB2IP coordinates both PI3K-Akt and ASK1 pathways for cell survival and apoptosis. Proc Natl Acad Sci USA 106: 19878-19883, 2009.

17. Yu L, Tumati V, Tseng SF, Hsu FM, Kim DN, Hong D, Hsieh JT, Jacobs C, Kapur P and Saha D: DAB2IP regulates autophagy in prostate cancer in response to combined treatment of radiation and a DNA-PKcs inhibitor. Neoplasia 14: 1203-1212, 2012.

18. KongZ, Xie D, Boike T, Raghavan P,BurmaS, Chen DJ,Habib AA, Chakraborty A, Hsieh JT and Saha D: Downregulation of human DAB2IP gene expression in prostate cancer cells results in resistance to ionizing radiation. Cancer Res 70: 2829-2839, 2010.

19. Xie D, Gore C, Liu J, Pong RC, Mason R, Hao G, Long M, Kabbani W, Yu L, Zhang H, et al: Role of DAB2IP in modulating epithelial-to-mesenchymal transition and prostate cancer metastasis. Proc Natl Acad Sci USA 107: 2485-2490, 2010.

20. Yun EJ, Baek ST, Xie D, Tseng SF, Dobin T, Hernandez E, Zhou J, Zhang L, Yang J, Sun H, et al: DAB2IP regulates cancer stem cell phenotypes through modulating stem cell factor receptor and ZEB1. Oncogene 34: 2741-2752, 2015.

21. Cancer Genome Atlas Research Network: Comprehensive molecular characterization of urothelial bladder carcinoma. Nature 507: 315-322, 2014.

22. Choi W,Porten S,KimS, Willis D,PlimackER,Hoffman-Censits J, Roth B, Cheng T, Tran M, Lee IL, et al: Identification of distinct basal and luminal subtypes of muscle-invasive bladder cancer with different sensitivities to frontline chemotherapy. Cancer Cell 25: 152-165, 2014.

23. Zhang T, Fan J, Wu K, Zeng J, Sun K, Guan Z, Wang X, Hiesh JT and He D: Roles of HIF-1 $\alpha$ in a novel optical orthotopic spontaneous metastatic bladder cancer animal model. Urol Oncol 30: 928-935, 2012.

24. Wu K, Zeng J, Zhou J, Fan J, Chen Y, Wang Z, Zhang T, Wang X and He D: Slug contributes to cadherin switch and malignant progression in muscle-invasive bladder cancer development. Urol Oncol 31: 1751-1760, 2012.

25. Wu K, Fan J, Zhang L, Ning Z, Zeng J, Zhou J, Li L, Chen Y, Zhang T, Wang X, et al: PI3K/Akt to GSK3 $\beta / \beta$-catenin signaling cascade coordinates cell colonization for bladder cancer bone metastasis through regulating ZEB1 transcription. Cell Signal 24: 2273-2282, 2012.

26. Blick C, Ramachandran A, Wigfield S, McCormick R, Jubb A, Buffa FM, Turley H, Knowles MA, Cranston D, Catto J, et al: Hypoxia regulates FGFR3 expression via HIF-1 $\alpha$ and miR-100 and contributes to cell survival in non-muscle invasive bladder cancer. Br J Cancer 109: 50-59, 2013.

27. Chen H, Pong RC, Wang Z and Hsieh JT: Differential regulation of the human gene DAB2IP in normal and malignant prostatic epithelia: Cloning and characterization. Genomics 79: 573-581, 2002.

28. Dote H, Toyooka S, Tsukuda K, Yano M, Ouchida M, Doihara H, Suzuki M, Chen H, Hsieh JT, Gazdar AF, et al: Aberrant promoter methylation in human DAB2 interactive protein (hDAB2IP) gene in breast cancer. Clin Cancer Res 10: 2082-2089, 2004.

29. Yano M, Toyooka S, Tsukuda K, Dote H, Ouchida M, Hanabata T, Aoe M, Date H, Gazdar AF and Shimizu N: Aberrant promoter methylation of human DAB2 interactive protein (hDAB2IP) gene in lung cancers. Int J Cancer 113: 59-66, 2005.

30. Dote H, Toyooka S, Tsukuda K, Yano M, Ota T, Murakami M, Naito M, Toyota M, Gazdar AF and Shimizu N: Aberrant promoter methylation in human DAB2 interactive protein (hDAB2IP) gene in gastrointestinal tumour. Br J Cancer 92: 1117-1125, 2005

31. Calvisi DF, Ladu S, Conner EA, Seo D, Hsieh JT, Factor VM and Thorgeirsson SS: Inactivation of Ras GTPase-activating proteins promotes unrestrained activity of wild-type Ras in human liver cancer. J Hepatol 54: 311-319, 2011. 
32. Duan YF, Li DF, Liu YH, Mei P, Qin YX, Li LF, Lin QX and Li ZJ: Decreased expression of DAB2IP in pancreatic cancer with wild-type KRAS. Hepatobiliary Pancreat Dis Int 12: 204-209, 2013

33. Smits M, van Rijn S, Hulleman E, Biesmans D, van Vuurden DG, Kool M, Haberler C, Aronica E, Vandertop WP, Noske DP, et al: EZH2-regulated DAB2IP is a medulloblastoma tumor suppressor and a positive marker for survival. Clin Cancer Res 18 4048-4058, 2012.

34. Xu Y, He J, Wang Y, Zhu X, Pan Q, Xie Q and Sun F: miR-889 promotes proliferation of esophageal squamous cell carcinomas through DAB2IP. FEBS Lett 589: 1127-1135, 2015.

35. Chen H, Tu SW and Hsieh JT: Down-regulation of human DAB2IP gene expression mediated by polycomb Ezh2 complex and histone deacetylase in prostate cancer. J Biol Chem 280 22437-22444, 2005.

36. Tsai YS, Lai CL, Lai CH, Chang KH, Wu K, Tseng SF, Fazli L, Gleave M, Xiao G, Gandee L, et al: The role of homeostatic regulation between tumor suppressor DAB2IP and oncogenic Skp2 in prostate cancer growth. Oncotarget 5: 6425-6436, 2014.
37. Liao H, Xiao Y, Hu Y, Xiao Y, Yin Z and Liu L: microRNA-32 induces radioresistance by targeting DAB2IP and regulating autophagy in prostate cancer cells. Oncol Lett 10: 2055-2062, 2015.

38. Haug BH, Henriksen JR, Buechner J, Geerts D, Tømte E, Kogner P, Martinsson T, Flægstad T, Sveinbjørnsson B and Einvik C: MYCN-regulated miRNA-92 inhibits secretion of the tumor suppressor DICKKOPF-3 (DKK3) in neuroblastoma. Carcinogenesis 32: 1005-1012, 2011.

39. Li Y, Li L, Guan Y, Liu X, Meng Q and Guo Q: MiR-92b regulates the cell growth, cisplatin chemosensitivity of A549 non small cell lung cancer cell line and target PTEN. Biochem Biophys Res Commun 440: 604-610, 2013.

40. Qian NS, Liu WH, Lv WP, Xiang X, Su M, Raut V, Chen YL and Dong JH: Upregulated microRNA-92b regulates the differentiation and proliferation of EpCAM-positive fetal liver cells by targeting C/EBPß. PLoS One 8: e68004, 2013. 